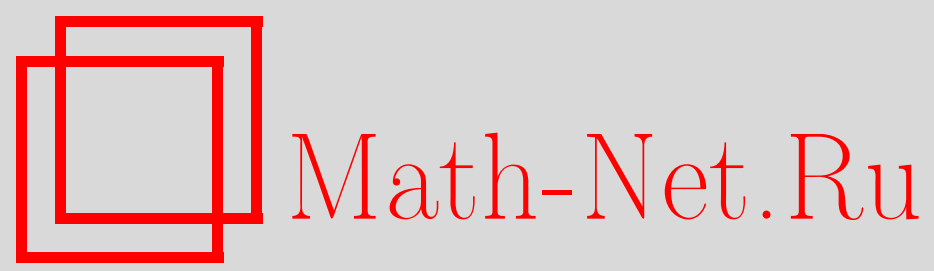

О. М. Мартынов, О сильной единственности минимальных проекций в пространстве $l_{\infty}^{9}$, Вестник ТвГУ. Серия: Прикладная математика, 2020, выпуск 4, 28-42

DOI: https://doi.org/10.26456/vtpmk603

Использование Общероссийского математического портала Math-Net.Ru подразумевает, что вы прочитали и согласны с пользовательским соглашением http: //www. mathnet.ru/rus/agreement

Параметры загрузки:

IP : 3.81 .55 .215

26 апреля 2023 г., $18: 16: 24$ 
УДК 513.88

\section{О СИЛЬНОЙ ЕДИНСТВЕННОСТИ МИНИМАЛЬНЫХ ПРОЕКЦИЙ В ПРОСТРАНСТВЕ $l_{\infty}^{9}$}

\section{Мартынов О.M.}

Военная академия воздушно-космической обороны имени Маршала Советского

Союза Г.К. Жукова, г. Тверь

Поступила в редакчию 30.09.2020, после переработки 23.10.2020.

В работе рассматриваются минимальные проекции пространства $l_{\infty}^{9}$ на некоторые подпространства коразмерности 3. Для них найдены относительные прекционные константы, а в случае минимальной проекции с единичной нормой найдено максимальной значение константы сильной единственности. Найденые проекционные константы могут найти применение в вычислительной математике, в частности, для оценки сходимости проекционных методов решения операторных уравнений и в оценках ошибки алгоритма Ремеза.

Ключевые слова: пространство, подпространство, оператор проектирования (проекция), относительная проекционная константа, константа сильной единственности.

Вестник ТвГУ. Серия: Прикладнал математика. 2020. № 4. С. 28-42. https://doi.org/10.26456/vtpmk603

\section{1. Введение}

Пусть $Y$ - замкнутое подпространство банахова пространства $X$.

Определение 1. Линейный ограниченный оператор $\pi: X \rightarrow Y$ называется оператором проектирования (проекцией) пространства $X$ на $Y$, если $\pi y=y \partial л я$ любого $y \in Y$.

Множество всех операторов проектирования пространства $X$ на подпространство $Y$ будем обозначать через $\pi(X, Y)$.

Определение 2. Относительной проекиионной константой подпространства $Y$ в пространстве $X$ называется число $\lambda(Y, X)=\inf \{\|\pi\|, \pi \in \pi(X, Y)\}$.

Среди операторов проектирования особый интерес представляют те, для которых выполняется равенство $\|\pi\|=\lambda(Y, X)$. Такие проекции, если они существуют, называются минимальными.

Множество минимальных проекций из $X$ на $Y$ будем обозначать $\Delta(X, Y)$.

Определение 3. Если множество $\Delta(X, Y)$ состоит ровно из одного элемента, то говорлт, что пара $(X, Y)$ обладает свойством единственности. 
Условия единственности для некоторых минимальных проекций в пространствах $l_{\infty}^{n}$ и $l_{1}^{n}$ можно найти, например, в [1], [5], [9], [17] и [20].

Пусть $Y_{6}$ есть 6-мерное подпространство пространства $X=l_{\infty}^{9}, 9$-мерного линейного нормированного пространства элементов $x=\left(x_{i}\right)_{1}^{9}$ с нормой

$$
\|x\|=\max _{1 \leqslant i \leqslant 9}\left|x_{i}\right| .
$$

Предложение 1. [1] Любой оператор проектирования $\pi: l_{\infty}^{9} \rightarrow Y_{6}$ имеет вид $\pi_{\alpha}$, где

$$
\pi_{\alpha} x=x-\sum_{i=1}^{3} \alpha_{i} f_{i}(x)
$$

$\alpha_{i}=\left(\alpha_{i j}\right)_{j=1}^{9}$ - элементы пространства $l_{\infty}^{9}, a f_{i}=\left(f_{i j}\right)_{j=1}^{9} \neq 0$ - линейнъе функционалы, определенные в $l_{\infty}^{9}$, причем

$$
f_{i}\left(\alpha_{j}\right)=\sum_{k=1}^{9} \alpha_{j k} f_{i k}=\delta_{i j}(i, j=1,2,3) .
$$

Гиперплоскости пространства $l_{\infty}^{9}$ имеют вид

$$
f_{i}^{-1}(0)=\left\{x \in l_{\infty}^{9} \mid f_{i}(x)=\sum_{k=1}^{9} f_{i k} x_{k}=0\right\}(i=1,2,3),
$$

и в силу линейной независимости функционалов $f_{i}(i=1,2,3)$, которая следует из формул (1.1), пространство $Y_{6}=\cap_{i=1}^{3} f_{i}^{-1}(0)$ является подпространством пространства $l_{\infty}^{9}$ коразмерности 3 .

Определение 4. Оператор проектирования $\pi_{0} \in \Delta\left(l_{\infty}^{9}, Y_{6}\right)$ называется сильно единственным, если для любого $\pi \in \pi\left(l_{\infty}^{9}, Y_{6}\right)$ справедливо неравенство

$$
\left\|\pi_{0}\right\|+k \cdot\left\|\pi-\pi_{0}\right\| \leqslant\|\pi\|
$$

где $k$ не зависит от $\pi u k \in(0 ; 1]$.

Число $k$ называется константой сильной единственности, а через $k_{0}$ обозначается максимальное значение $k$, при котором выполняется неравенство (1.2).

Очевидно, что сильно единственный оператор $\pi_{0}$ имеет минимальную норму, а пара $\left(l_{\infty}^{9}, Y_{6}\right)$ обладает свойством единственности.

Предложение 2. [1] Нормы операторов $\pi u \pi-\pi_{0}$ вычисляются по бормулам

$$
\|\pi\|=\max _{1 \leqslant i \leqslant 9} T_{i},\left\|\pi-\pi_{0}\right\|=\max _{1 \leqslant i \leqslant 9} B_{i}
$$

əวe

$$
T_{i}=\sum_{j=1}^{9}\left|\delta_{i j}-\sum_{k=1}^{3} \alpha_{k i} f_{k j}\right|, B_{i}=\sum_{j=1}^{9}\left|\sum_{k=1}^{3}\left(\alpha_{k i}-\alpha_{k i}^{(0)}\right) f_{k j}\right|
$$

а оператор $\pi_{0}$ имеет вид $\pi_{\alpha}^{(0)}$, где

$$
\pi_{\alpha}^{(0)} x=x-\sum_{k=1}^{3} \alpha_{k}^{(0)} f_{k}(x)
$$


Определение 5. Абсолютной проекиионной константой порядков $k, n$ называется число

$$
\lambda(k, n)=\sup \left\{\lambda\left(Y_{k}, X_{n}\right): Y_{k} \subset X_{n}\right\},
$$

где $X_{n}$ и $Y_{k}$ - любые пространства размерности $n$ и их подпространства размерности $k$ сответственно, $k, n \in \mathbb{N}, k<n$.

Как отмечено в [16], теория аппроксимации является как «заказчиком», так и «поставщиком» новых идей при изучении минимальных проекций и проекционных констант. В частности, с теорией аппроксимации связано появление понятия сильной единственности, введенное Ньюменом и Шапиро в 1963-64 годах ( [14], [15]).

Напомним, что элемент $v_{0} \in V \subset Z$ называется сильно единственным элементом наилучшего приближения к $z \in Z$, если существует вещественное число $k>0$ такое, что для любого $v \in V$ справедливо неравенство

$$
\left\|z-v_{0}\right\|+k \cdot\left\|v-v_{0}\right\| \leqslant\|z-v\| .
$$

Если в этом определении положить $Z=\pi\left(l_{\infty}^{9}, Y_{6}\right), z=0, v=\pi$ и $v_{0}=\pi_{0}$, то придем к определению 4.

Вычисление проекционных констант (или их оценок) находит применение в вычислительной математике. В частности, для оценки сходимости проекционных методов решения линейных и нелинейных операторных уравнений удобно использовать абсолютные проекционные константы $\lambda(k, n)$ или их оценки. Известно [20], что суть проекционных методов состоит в следующем. Пусть $X$ - бесконечномерное банахово пространство, $Y$ - другое банахово пространство. Пусть $A$ - линейный (вообще говоря, неограниченный) оператор с областью определения $D(A) \subset X$ и областью значений $\operatorname{Im}(A) \subset Y$. Пусть также заданы две последовательности подпространств $\left(X_{n}\right)$ и $\left(Y_{n}\right): X_{n} \subset D(A), Y_{n} \subset Y(n=1,2, \ldots)$ и последовательность операторов проектирования: $\left(\pi_{n}\right): Y \rightarrow Y_{n}, \pi_{n}(Y)=Y_{n}(n=1,2, \ldots)$. Пусть дано линейное уравнение $A x=y$ (здесь $x \in D(A), y \in \operatorname{Im}(A))$. Заменим это уравнение приближенным: $\pi_{n}\left(A x_{n}-y\right)=0$, где $x_{n} \in X_{n}$. Отыскание последовательности $\left(x_{n}\right)$ и выяснение ее сходимости и составляет суть проекционных методов.

$\mathrm{K}$ числу проекционных методов относят, например, метод Петрова-Галеркина, метод Ритца, а также метод конечных элементов. Последний можно рассматривать как синтез методов Петрова-Галеркина и конечньх разностей. Весьма упрощенно можно сказать, что метод конечных элементов это проекционный метод со специальными координатными функциями, заменяющими базис $\left(e_{i}\right)_{1}^{\infty}$.

Константы сильной единственности нашли широкое применение в оценках ошибки алгоритма Ремеза [18].

При изучении минимальных проекций ставятся две проблемы: 1) вопрос о существовании таких проекций; 2) вопрос об их единственности.

Для конечномерного пространства $X$ актуальной является как проблема единственности минимальной проекции, так и определения точных значений $\lambda(Y, X)$ или их оценок сверху или снизу.

Для $\lambda(k, n)$ известны следующие точные значения:

$-\lambda(1, n)=1[20]$

$-\lambda(n-1, n)=\frac{2(n-1)}{n}[2]$, 
$-\lambda(3,5)=\frac{5+4 \sqrt{2}}{7}[3]$.

Известны [4] оценки для $\lambda(k, n)$ сверху, а именно

$$
\lambda(k, n) \leqslant \varphi(k, n)=\frac{k+\sqrt{k(n-1)(n-k)}}{n} .
$$

Что касается оценок снизу, то они известны лишь для частных случаев и получены, в основном, с помощью относительных проекционных констант. При этом точность полученных оценок можно определить с помощью неравенств

$$
\lambda\left(Y_{n-k}, l_{\infty}^{n}\right) \leqslant \lambda(n-k, n) \leqslant \varphi(n-k, n), \lambda\left(Y_{n-k}, l_{1}^{n}\right) \leqslant \lambda(n-k, n) \leqslant \varphi(n-k, n) .
$$

В работах [1], [5] определены относительные проекционные константы гиперплоскостей в пространствах $l_{1}, l_{1}^{n}, l_{\infty}, l_{\infty}^{n}$. В работе Локтя [7] были получены оценки снизу для $\lambda(n-2, n)$ и $\lambda(n-3, n)$. Для получения этих оценок были найдены относительные проекционные константы $\lambda\left(Y_{n-2}, l_{1}^{n}\right), \lambda\left(Y_{n-2}, l_{\infty}^{n}\right), \lambda\left(Y_{n-3}, l_{1}^{n}\right)$ для некоторых классов подпространств. Дальнейшее развитие этих результатов для оценок снизу констант $\lambda(n-4, n)$ и $\lambda(n-5, n)$ можно найти в [9].

$\mathrm{C}$ проблемой единственности тесно связана проблема сильной единственности минимальных проекций.

В [5] Левицким было найдено максимальное значение константы сильной единственности операторов проектирования с единичной нормой на гиперплоскость в пространствах $l_{\infty}^{n}$ и $l_{1}^{n}$. Там же получены некоторые оценки констант сильной единственности операторов проектирования с нормой, большей единицы, опять же для случая гиперплоскости. Локтем [8] получено максимальное значение константы сильной единственности оператора проектирования с неединичной нормой на гиперплоскость в пространстве $l_{\infty}^{n}$. В работах [10], [11] найдены максимальные значения констант сильной единственности проекций на подпространства коразмерности 2 и 3 пространства $l_{\infty}^{4}$. Позднее результат работы [11] был обобщен для пространств размерности $n \geqslant 3$ [19], а дальнейшее продвижение было получено в [6]. Также, в [12], [13] были найдены константы сильной единственности для операторов проектирования с единичной нормой на некоторые подпространства коразмерности 2 в пространстве $l_{\infty}^{2 n}$.

Найдем проекционные константы для операторов проектирования пространства $l_{\infty}^{9}$ на некоторый класс подпространств коразмерности 3.

\section{2. Относительные проекционные константы}

Функционалы $f_{i}(i=1,2,3)$ зададим следующим образом:

$$
f_{i}=\left(f_{i j}\right)_{j=1}^{9}, \text { где } f_{i j}=\left\{\begin{array}{c}
1, \text { если } j=i, j=i+6 \\
r, \text { если } j=i+3 \\
0, \text { если } j \neq i, j \neq i+3, j \neq i+6 .
\end{array}\right.
$$

где $r>0$. В этом случае, если $Y_{6}=\cap_{i=1}^{3} f_{i}^{-1}(0)$, то для проекций $\pi_{\alpha} \in \pi\left(l_{\infty}^{9}, Y_{6}\right)$ соотношения (1.1) примут вид

$$
f_{i}\left(\alpha_{j}\right)=\alpha_{j i}+r \alpha_{j i+3}+\alpha_{j i+6}=\delta_{i j}(i, j=1,2,3) .
$$


Теорема 1. Пусть $\pi_{\alpha}^{(0)}$-минималъный оператор проектирования пространства $l_{\infty}^{9}$ на подпространство $Y_{6}$, определяемое функиионалами (2.1). Тогда

$$
\text { (1) } \lambda\left(Y_{6}, l_{\infty}^{9}\right)=\left\|\pi_{\alpha}^{(0)}\right\|=1, \text { eсли } r \geqslant 2
$$

(2) $\lambda\left(Y_{6}, l_{\infty}^{9}\right)=\left\|\pi_{\alpha}^{(0)}\right\|=\frac{4}{r^{2}-2 r+4}$, ecлu $0<r<2$

Доказательство. Найдем значения $T_{i}^{(0)}=\sum_{j=1}^{9}\left|\delta_{i j}-\sum_{k=1}^{3} \alpha_{k i}^{(0)} f_{k j}\right|(i=1, \cdots, 9)$. Имеем

$$
\begin{gathered}
T_{1}^{(0)}=\sum_{j=1}^{9}\left|\delta_{1 j}-\sum_{k=1}^{3} \alpha_{k 1}^{(0)} f_{k j}\right|=\left|1-\alpha_{11}^{(0)}\right|+(1+r)\left|\alpha_{11}^{(0)}\right|+(2+r)\left(\left|\alpha_{21}^{(0)}\right|+\left|\alpha_{31}^{(0)}\right|\right), \\
T_{2}^{(0)}=\left|1-\alpha_{22}^{(0)}\right|+(1+r)\left|\alpha_{22}^{(0)}\right|+(2+r)\left(\left|\alpha_{12}^{(0)}\right|+\left|\alpha_{32}^{(0)}\right|\right), T_{3}^{(0)}=\left|1-\alpha_{33}^{(0)}\right|+(1+r)\left|\alpha_{33}^{(0)}\right| \\
+(2+r)\left(\left|\alpha_{13}^{(0)}\right|+\left|\alpha_{23}^{(0)}\right|\right), T_{4}^{(0)}=\left|1-r \alpha_{14}^{(0)}\right|+2\left|\alpha_{14}^{(0)}\right|+(2+r)\left(\left|\alpha_{24}^{(0)}\right|+\left|\alpha_{34}^{(0)}\right|\right), \\
T_{5}^{(0)}=\left|1-r \alpha_{25}^{(0)}\right|+2\left|\alpha_{25}^{(0)}\right|+(2+r)\left(\left|\alpha_{15}^{(0)}\right|+\left|\alpha_{35}^{(0)}\right|\right), T_{6}^{(0)}=\left|1-r \alpha_{36}^{(0)}\right|+2\left|\alpha_{36}^{(0)}\right| \\
+(2+r)\left(\left|\alpha_{16}^{(0)}\right|+\left|\alpha_{26}^{(0)}\right|\right), T_{7}^{(0)}=\left|1-\alpha_{17}^{(0)}\right|+(1+r)\left|\alpha_{17}^{(0)}\right|+(2+r)\left(\left|\alpha_{27}^{(0)}\right|+\left|\alpha_{37}^{(0)}\right|\right), \\
T_{8}^{(0)}=\left|1-\alpha_{28}^{(0)}\right|+(1+r)\left|\alpha_{28}^{(0)}\right|+(2+r)\left(\left|\alpha_{18}^{(0)}\right|+\left|\alpha_{38}^{(0)}\right|\right), T_{9}^{(0)}=\left|1-\alpha_{39}^{(0)}\right|+(1+r)\left|\alpha_{39}^{(0)}\right| \\
+(2+r)\left(\left|\alpha_{19}^{(0)}\right|+\left|\alpha_{29}^{(0)}\right|\right) .
\end{gathered}
$$

Рассмотрим два случая.

(1) $r \geqslant 2$. В равенствах, полученных для $T_{i}(i=1, \cdots, 9)$, положим $\alpha_{14}^{(0)}=\alpha_{25}^{(0)}=\alpha_{36}^{(0)}=\frac{1}{r}$, все остальные $\alpha_{i j}$ пусть будут равны нулю. При этом условия (2.2) выполняются. Тогда $T_{1}^{(0)}=T_{2}^{(0)}=T_{3}^{(0)}=T_{7}^{(0)}=T_{8}^{(0)}=T_{9}^{(0)}=1, T_{4}^{(0)}=T_{5}^{(0)}=T_{6}^{(0)}=\frac{2}{r} \leqslant 1$.

Следовательно,

$$
\lambda\left(Y_{6}, l_{\infty}^{9}\right)=\left\|\pi_{\alpha}^{(0)}\right\|=\max _{1 \leqslant i \leqslant 9} T_{i}^{(0)}=1 .
$$

(2) $0<r<2$. Очевидно, что $\left\|\pi_{\alpha}^{(0)}\right\| \geqslant T_{i}^{(0)}(i=1, \cdots, 9)$. Оценим выражения для $T_{i}^{(0)}(i=1, \cdots, 9)$ снизу. Для этого воспользуемся тем, что $|x-y| \geqslant|x|-|y|$ и очевидными неравенствами $\left|\alpha_{i j}^{(0)}\right| \geqslant 0$. Тогда

$$
\left\|\pi_{\alpha}^{(0)}\right\| \geqslant T_{1}^{(0)} \geqslant 1-\left|\alpha_{11}^{(0)}\right|+(1+r)\left|\alpha_{11}^{(0)}\right|=1+r\left|\alpha_{11}\right| .
$$

Аналогично получим

$$
\begin{gathered}
\left\|\pi_{\alpha}^{(0)}\right\| \geqslant T_{2}^{(0)} \geqslant 1+r\left|\alpha_{22}\right|,\left\|\pi_{\alpha}^{(0)}\right\| \geqslant T_{3}^{(0)} \geqslant 1+r\left|\alpha_{33}\right|,\left\|\pi_{\alpha}^{(0)}\right\| \geqslant T_{4}^{(0)} \geqslant 1+(2-r)\left|\alpha_{14}\right|, \\
\left\|\pi_{\alpha}^{(0)}\right\| \geqslant T_{5}^{(0)} \geqslant 1+(2-r)\left|\alpha_{25}\right|,\left\|\pi_{\alpha}^{(0)}\right\| \geqslant T_{6}^{(0)} \geqslant 1+(2-r)\left|\alpha_{36}\right|, \\
\left\|\pi_{\alpha}^{(0)}\right\| \geqslant T_{7}^{(0)} \geqslant 1+r\left|\alpha_{17}\right|,\left\|\pi_{\alpha}^{(0)}\right\| \geqslant T_{8}^{(0)} \geqslant 1+r\left|\alpha_{28}\right|,\left\|\pi_{\alpha}^{(0)}\right\| \geqslant T_{9}^{(0)} \geqslant 1+r\left|\alpha_{39}\right| .
\end{gathered}
$$


Из соотношений (2.2) следует, что

$$
\alpha_{14}=\frac{1}{r}\left(1-\alpha_{11}^{(0)}-\alpha_{17}^{(0)}\right), \alpha_{25}=\frac{1}{r}\left(1-\alpha_{22}^{(0)}-\alpha_{28}^{(0)}\right), \alpha_{36}=\frac{1}{r}\left(1-\alpha_{33}^{(0)}-\alpha_{39}^{(0)}\right) .
$$

Используя эти равенства, умножим неравенства для $T_{i}^{(0)}(i=4,5,6)$ на $\frac{r^{2}}{2-r}>0$ и сложим все неравенства для $T_{i}^{(0)}(i=1, \cdots, 9)$ почленно. Получим

$$
\begin{gathered}
\left(6+\frac{3 r^{2}}{2-r}\right)\left\|\pi_{\alpha}^{(0)}\right\| \geqslant 6+\frac{3 r^{2}}{2-r}+r\left(\left|\alpha_{11}^{(0)}\right|+\left|\alpha_{22}^{(0)}\right|+\left|\alpha_{33}^{(0)}\right|+\left|\alpha_{17}^{(0)}\right|+\left|\alpha_{28}^{(0)}\right|+\left|\alpha_{39}^{(0)}\right|\right) \\
+r^{2}\left(\left|\alpha_{14}^{(0)}\right|+\left|\alpha_{25}^{(0)}\right|+\left|\alpha_{36}^{(0)}\right|\right)=6+\frac{3 r^{2}}{2-r}+r\left(\left|\alpha_{11}^{(0)}\right|+\left|\alpha_{22}^{(0)}\right|+\left|\alpha_{33}^{(0)}\right|+\left|\alpha_{17}^{(0)}\right|+\left|\alpha_{28}^{(0)}\right|\right. \\
\left.+\left|\alpha_{39}^{(0)}\right|\right)+r\left(\left|1-\alpha_{11}^{(0)}-\alpha_{17}^{(0)}\right|+\left|1-\alpha_{22}^{(0)}-\alpha_{28}^{(0)}\right|+\left|1-\alpha_{33}^{(0)}-\alpha_{39}^{(0)}\right|\right) \geqslant 6+\frac{3 r^{2}}{2-r} \\
+r\left(\left|\alpha_{11}^{(0)}\right|+\left|\alpha_{22}^{(0)}\right|+\left|\alpha_{33}^{(0)}\right|+\left|\alpha_{17}^{(0)}\right|+\left|\alpha_{28}^{(0)}\right|+\left|\alpha_{39}^{(0)}\right|\right)+r\left(3-\left|\alpha_{11}^{(0)}\right|-\left|\alpha_{17}^{(0)}\right|-\left|\alpha_{22}^{(0)}\right|\right. \\
\left.-\left|\alpha_{28}^{(0)}\right|-\left|\alpha_{33}^{(0)}\right|-\left|\alpha_{39}^{(0)}\right|\right)=6+\frac{3 r^{2}}{2-r}+3 r=\frac{12}{2-r} .
\end{gathered}
$$

Таким образом,

$$
\left(2+\frac{r^{2}}{2-r}\right)\left\|\pi_{\alpha}^{(0)}\right\| \geqslant \frac{4}{2-r} .
$$

Следовательно,

$$
\lambda\left(Y_{6}, l_{\infty}^{9}\right)=\left\|\pi_{\alpha}^{(0)}\right\| \geqslant \frac{4}{r^{2}-2 r+4} .
$$

Покажем, что эта оценка снизу точная. Для этого найдем $\alpha_{i}^{(0)}(i=1,2,3)$ такие, что

$$
T_{i}^{(0)}=\frac{4}{r^{2}-2 r+4}(i=1, \ldots, 9) .
$$

Положим

$$
\begin{gathered}
\alpha_{14}^{(0)}=\alpha_{25}^{(0)}=\alpha_{36}^{(0)}=\frac{r}{r^{2}-2 r+4} \\
\alpha_{11}^{(0)}=\alpha_{17}^{(0)}=\alpha_{22}^{(0)}=\alpha_{28}^{(0)}=\alpha_{33}^{(0)}=\alpha_{39}^{(0)}=\frac{2-r}{r^{2}-2 r+4} .
\end{gathered}
$$

Остальные $\alpha_{i j}$ положим равными нулю. Условия (2.2) при этом выполняются.

Тогда

$$
\begin{gathered}
T_{1}^{(0)}=T_{2}^{(0)}=T_{3}^{(0)}=T_{7}^{(0)}=T_{8}^{(0)}=T_{9}^{(0)}=\left|1-\alpha_{11}^{(0)}\right|+(1+r)\left|\alpha_{11}^{(0)}\right|=\left|1-\frac{2-r}{r^{2}-2 r+4}\right| \\
+\frac{(1+r)(2-r)}{r^{2}-2 r+4}=\frac{r^{2}-r+2}{r^{2}-2 r+4}+\frac{2+r-r^{2}}{r^{2}-2 r+4}=\frac{4}{r^{2}-2 r+4}, \\
T_{4}^{(0)}=T_{5}^{(0)}=T_{6}^{(0)}=\left|1-r \alpha_{14}^{(0)}\right|+2\left|\alpha_{14}^{(0)}\right|=\left|1-\frac{r^{2}}{r^{2}-2 r+4}\right|+\frac{2 r}{r^{2}-2 r+4} \\
=\frac{4-2 r}{r^{2}-2 r+4}+\frac{2 r}{r^{2}-2 r+4}=\frac{4}{r^{2}-2 r+4} .
\end{gathered}
$$


Таким образом,

$$
\lambda\left(Y_{6}, l_{\infty}^{9}\right)=\left\|\pi_{\alpha}^{(0)}\right\|=\frac{4}{r^{2}-2 r+4} .
$$

Очевидно, что $\frac{4}{r^{2}-2 r+4}>1$, так как это неравенство равносильно условию $0<r<2$.

Пусть теперь функционалы $f_{i}(i=1,2,3)$ имеют вид :

$$
f_{i}=\left(f_{i j}\right)_{j=1}^{9}, \text { где } f_{i j}=\left\{\begin{array}{c}
r, \text { если } j=i, j=i+6 \\
1, \text { если } j=i+3 \\
0, \text { если } j \neq i, j \neq i+3, j \neq i+6 .
\end{array}\right.
$$

где $r>0$. Тогда справедливо следующее утверждение.

Теорема 2. Пусть $\tilde{\pi}_{\alpha}^{(0)} \tilde{\widetilde{Y}}_{6}$ минималъный оператор проектирования пространства $l_{\infty}^{9}$ на подпространство $\widetilde{Y}_{6}$, определяемое функиионалами (2.3). Тогда

$$
\text { (1) } \lambda\left(\widetilde{Y}_{6}, l_{\infty}^{9}\right)=\left\|\widetilde{\pi}_{\alpha}^{(0)}\right\|=1, \text { если } 0<r \leqslant \frac{1}{2} ;
$$

(2) $\lambda\left(\widetilde{Y}_{6}, l_{\infty}^{9}\right)=\left\|\widetilde{\pi}_{\alpha}^{(0)}\right\|=\frac{4 r^{2}}{4 r^{2}-2 r+1}$, если $r>\frac{1}{2}$.

Доказательство. Для доказательства утверждения теоремы 2 можно провести рассуждения аналогичные тем, которые были приведены при доказательстве теоремы 1. Поступим иначе. Рассмотрим функционалы $\widehat{f}_{i}(i=1,2,3)$, которые определяются так:

$$
\widehat{f}_{i}=\frac{f_{i}}{r}=\left(\widehat{f}_{i j}\right)_{j=1}^{9}, \text { где } \widehat{f}_{i j}=\left\{\begin{array}{c}
1, \text { если } j=i, j=i+6 \\
1 / r, \text { если } j=i+3 \\
0, \text { если } j \neq i, j \neq i+3, j \neq i+6 .
\end{array}\right.
$$

Очевидно, что подпространство $\widehat{Y}_{6}=\cap_{i=1}^{3} \widehat{f}_{i}^{-1}(0)$ совпадает с подпространством $\widetilde{Y}_{6}$ и при $s=1 / r$ для функционалов (2.4) справедлива теорема 1. Это значит, что

$$
\text { (1) } \lambda\left(\widehat{Y}_{6}, l_{\infty}^{9}\right)=1 \text {, если } s \geqslant 2 ;(2) \lambda\left(\widehat{Y}_{6}, l_{\infty}^{9}\right)=\frac{4}{s^{2}-2 s+4} \text {, если } 0<s<2 .
$$

Заменив в полученных константах $s$ на $1 / r$, получим относительные проекционные константы из условия теоремы 2.

\section{3. Константы сильной единственности}

Лемма 1. Значение константы сильной единствености $k$ из неравенства (1.2) для оператора проектирования $\pi_{\alpha}^{(0)}$, который определяется функционалами (2.1), удовлетворяет условию

$$
k \leqslant \frac{r}{r+2},
$$

если $r \geqslant 2$. 
Доказательство. Для получения оценки константы $k$ сверху рассмотрим оператор $\bar{\pi} x=x-\sum_{k=1}^{3} \bar{\alpha}_{k} f_{k}(x)$. Значения $\bar{\alpha}_{k}(k=1,2,3)$ определим следующим образом: $0<\bar{\alpha}_{11} \leqslant 1, \bar{\alpha}_{11}=\bar{\alpha}_{22}=\bar{\alpha}_{33}=\bar{\alpha}_{17}=\bar{\alpha}_{28}=\bar{\alpha}_{39}, \bar{\alpha}_{14}=\bar{\alpha}_{25}=\bar{\alpha}_{36} \geqslant 0$. Значения всех остальных $\alpha_{i j}$ положим равными нулю.

Вычислим нормы операторов $\bar{\pi}$ и $\bar{\pi}-\pi^{(0)}$.

Имеем

$$
\|\bar{\pi}\|=\max _{1 \leqslant i \leqslant 9} \bar{T}_{i}=\max _{i} \sum_{j=1}^{9}\left|\delta_{i j}-\sum_{k=1}^{3} \bar{\alpha}_{k i} f_{k j}\right| .
$$

Найдем значения $\bar{T}_{i}$, учитывая, что $1-\bar{\alpha}_{11} \geqslant 0,1-r \bar{\alpha}_{14}=\bar{\alpha}_{11}+\bar{\alpha}_{17}>0$.

$$
\begin{gathered}
\bar{T}_{1}=\bar{T}_{2}=\bar{T}_{3}=\bar{T}_{7}=\bar{T}_{8}=\bar{T}_{9}=1-\bar{\alpha}_{11}+(1+r) \bar{\alpha}_{11}=1+r \bar{\alpha}_{11}>1 \\
\bar{T}_{4}=\cdots=\bar{T}_{5}=\bar{T}_{6}=1-r \bar{\alpha}_{14}+2 \bar{\alpha}_{14}=1-r \bar{\alpha}_{2}+(1+(n-2) r) \bar{\alpha}_{2} \\
=1+(2-r) \bar{\alpha}_{14} \leqslant 1 .
\end{gathered}
$$

Таким образом,

$$
\max _{1 \leqslant i \leqslant 9} \bar{T}_{i}=\bar{T}_{1}=1+r \bar{\alpha}_{11}
$$

Далее

$$
\begin{gathered}
\left\|\bar{\pi}-\pi_{\alpha}^{(0)}\right\|=\max _{1 \leqslant i \leqslant 9} \bar{B}_{i}=\max _{1 \leqslant i \leqslant 9} \sum_{j=1}^{9}\left|\sum_{k=1}^{3}\left(\bar{\alpha}_{k i}-\alpha_{k i}^{(0)}\right) f_{k j}\right| \\
=\max \left\{\sum_{j=1}^{9}\left|\bar{\alpha}_{11} f_{1 j}\right| ; \sum_{j=1}^{9}\left|\bar{\alpha}_{22} f_{2 j}\right| ; \sum_{j=1}^{9}\left|\bar{\alpha}_{33} f_{3 j}\right| ; \sum_{j=1}^{9}\left|\left(\bar{\alpha}_{14}-\frac{1}{r}\right) f_{1 j}\right| ;\right. \\
\sum_{j=1}^{9}\left|\left(\bar{\alpha}_{25}-\frac{1}{r}\right) f_{2 j}\right| ; \sum_{j=1}^{9}\left|\left(\bar{\alpha}_{36}-\frac{1}{r}\right) f_{3 j}\right| ; \sum_{j=1}^{9}\left|\bar{\alpha}_{17} f_{1 j}\right| ; \sum_{j=1}^{9}\left|\bar{\alpha}_{28} f_{2 j}\right| ; \\
\left.\sum_{j=1}^{9}\left|\bar{\alpha}_{39} f_{3 j}\right|\right\}=\max \left\{(r+2)\left|\bar{\alpha}_{11}\right| ;(r+2)\left|\bar{\alpha}_{22}\right| ;(r+2)\left|\bar{\alpha}_{33}\right| ;(r+2)\left|\bar{\alpha}_{14}-\frac{1}{r}\right|\right. \\
\left.(r+2)\left|\bar{\alpha}_{25}-\frac{1}{r}\right| ;(r+2)\left|\bar{\alpha}_{36}-\frac{1}{r}\right| ;(r+2)\left|\bar{\alpha}_{17}\right| ;(r+2)\left|\bar{\alpha}_{28}\right| ;(r+2)\left|\bar{\alpha}_{39}\right|\right\} \\
=(r+2) \cdot \max \left\{\left|\bar{\alpha}_{11}\right| ;\left|\bar{\alpha}_{14}-\frac{1}{r}\right|\right\}=(r+2) \cdot \max \left\{\left|\bar{\alpha}_{11}\right| ; \frac{1}{r}\left|1-r \bar{\alpha}_{14}\right|\right\} \\
=(r+2) \cdot \max \left\{\left|\bar{\alpha}_{11}\right| ; \frac{1}{r}\left|\bar{\alpha}_{11}+\bar{\alpha}_{17}\right|\right\}=(r+2) \cdot \max \left\{\left|\bar{\alpha}_{11}\right| ; \frac{2}{r}\left|\bar{\alpha}_{11}\right|\right\}=(r+2) \bar{\alpha}_{11},
\end{gathered}
$$

так как $r \geqslant 2$.

Найдем для $k$ оценку сверху. Неравенство (1.2) примет вид $1+k \cdot(r+2) \bar{\alpha}_{11} \leqslant 1+r \bar{\alpha}_{11}$, откуда получим, что $k \leqslant \frac{r}{r+2}$. Очевидно, что $k \in(0,1]$. 
Теорема 3. Оператор проектирования $\pi_{\alpha}^{(0)}$ пространства $l_{\infty}^{9}$ на подпространство $Y_{6}$, определяемое функиионалами (2.1), является сильно единственным и максимальное значение константы сильной единственности $k_{0}$ равно $\frac{r}{r+2}$, если $r \geqslant 2$.

Доказательство. Имеем

$$
\begin{gathered}
T_{1}=\sum_{j=1}^{9}\left|\delta_{1 j}-\sum_{k=1}^{3} \alpha_{k 1} f_{k j}\right|=\left|1-\alpha_{11}\right|+(1+r)\left|\alpha_{11}\right|+(2+r)\left(\left|\alpha_{21}\right|+\left|\alpha_{31}\right|\right), \\
T_{2}=\left|1-\alpha_{22}\right|+(1+r)\left|\alpha_{22}\right|+(2+r)\left(\left|\alpha_{12}\right|+\left|\alpha_{32}\right|\right), T_{3}=\left|1-\alpha_{33}\right|+(1+r)\left|\alpha_{33}\right| \\
+(2+r)\left(\left|\alpha_{13}\right|+\left|\alpha_{23}\right|\right), T_{4}=\left|1-r \alpha_{14}\right|+2\left|\alpha_{14}\right|+(2+r)\left(\left|\alpha_{24}\right|+\left|\alpha_{34}\right|\right), \\
T_{5}=\left|1-r \alpha_{25}\right|+2\left|\alpha_{25}\right|+(2+r)\left(\left|\alpha_{15}\right|+\left|\alpha_{35}\right|\right), T_{6}=\left|1-r \alpha_{36}\right|+2\left|\alpha_{36}\right| \\
+(2+r)\left(\left|\alpha_{16}\right|+\left|\alpha_{26}\right|\right), T_{7}=\left|1-\alpha_{17}\right|+(1+r)\left|\alpha_{17}\right|+(2+r)\left(\left|\alpha_{27}\right|+\left|\alpha_{37}\right|\right), \\
T_{8}=\left|1-\alpha_{28}\right|+(1+r)\left|\alpha_{28}\right|+(2+r)\left(\left|\alpha_{18}\right|+\left|\alpha_{38}\right|\right), T_{9}=\left|1-\alpha_{39}\right|+(1+r)\left|\alpha_{39}\right| \\
+(2+r)\left(\left|\alpha_{19}\right|+\left|\alpha_{29}\right|\right) .
\end{gathered}
$$

Далее найдем

$$
B_{i}=\sum_{j=1}^{9}\left|\sum_{k=1}^{3}\left(\bar{\alpha}_{k i}-\alpha_{k i}^{(0)}\right) f_{k j}\right|(i=1, \cdots, 9)
$$

Для $i=1,2,3,7,8,9$ получим $B_{i}=(2+r) \sum_{k=1}^{3}\left|\alpha_{k i}\right|$. Остальные $B_{i}$ будут иметь вид:

$$
\begin{gathered}
B_{4}=(2+r)\left(\left|\alpha_{14}-\frac{1}{r}\right|+\left|\alpha_{24}\right|+\left|\alpha_{34}\right|\right), B_{5}=(2+r)\left(\left|\alpha_{15}\right|+\left|\alpha_{25}-\frac{1}{r}\right|+\left|\alpha_{35}\right|\right), \\
B_{6}=(2+r)\left(\left|\alpha_{16}\right|+\left|\alpha_{26}\right|+\left|\alpha_{36}-\frac{1}{r}\right|\right) .
\end{gathered}
$$

Покажем, что $k_{0}=\frac{r}{r+2}(r \geqslant 2)$ - максимально возможное значение константы сильной единственности. Для этого надо доказать, что неравенство

$$
\left\|\pi_{\alpha}^{(0)}\right\|+k_{0} \cdot \max _{1 \leqslant i \leqslant 9} B_{i} \leqslant \max _{1 \leqslant i \leqslant 9} T_{i}
$$

выполняется при любых значениях $\alpha_{i},(i=1,2,3)$.

Сравним значения $B_{i}$. Докажем неравенство

$$
B_{1}+B_{7} \geqslant 2 B_{4} .
$$

Оно равносильно неравенству

$$
\sum_{k=1}^{3}\left|\alpha_{k 1}\right|+\sum_{k=1}^{3}\left|\alpha_{k 7}\right| \geqslant 2\left(\left|\alpha_{14}-\frac{1}{r}\right|+\left|\alpha_{24}\right|+\left|\alpha_{34}\right|\right) .
$$


Теперь достаточно доказать два неравенства:

$$
\sum_{k=2}^{3}\left|\alpha_{k 1}\right|+\sum_{k=2}^{3}\left|\alpha_{k 7}\right| \geqslant 2 \sum_{k=2}^{3}\left|\alpha_{k 4}\right| \text { и }\left|\alpha_{11}\right|+\left|\alpha_{17}\right| \geqslant 2\left|\alpha_{14}-\frac{1}{r}\right| .
$$

Так как $\left|\alpha_{k 1}\right|+\left|\alpha_{k 7}\right| \geqslant\left|\alpha_{k 1}+\alpha_{k 1}\right|,\left|\alpha_{k 4}\right|=(1 / r)\left|\alpha_{k 1}+\alpha_{k 1}\right|(k=2,3)$ и $2 / r \leqslant 1$, то

$$
\sum_{k=2}^{3}\left|\alpha_{k 1}\right|+\sum_{k=2}^{3}\left|\alpha_{k 7}\right| \geqslant \sum_{k=2}^{3}\left|\alpha_{k 1}+\alpha_{k 7}\right| \geqslant \frac{2}{r} \sum_{k=2}^{3}\left|\alpha_{k 1}+\alpha_{k 7}\right|=2 \sum_{k=2}^{3}\left|\alpha_{k 4}\right|,
$$

что доказывает первое неравенство. Для доказательства второго неравенства воспользуемся тем, что $\alpha_{11}+\alpha_{17}=1-r \alpha_{14}$. Получим

$$
\left|\alpha_{11}\right|+\left|\alpha_{17}\right| \geqslant\left|\alpha_{11}+\alpha_{17}\right|=\left|1-r \alpha_{14}\right|=r\left|\frac{1}{r}-\alpha_{14}\right| \geqslant 2\left|\alpha_{14}-\frac{1}{r}\right| .
$$

Таким образом, неравенство (3.2) доказано.

Если $B_{i}<B_{4}(i=1,7)$, то неравенство (3.2) не выполняется. Следовательно, или $B_{1} \geqslant B_{4}$, или $B_{7} \geqslant B_{4}$ (или справедливы оба неравенства). Значит,

$$
\max _{i=1,4,7} B_{i}=\max _{i=1,7} B_{i}
$$

Аналогично доказывается,что

$$
\max _{i=2,5,8} B_{i}=\max _{i=2,8} B_{i}, \max _{i=3,6,9} B_{i}=\max _{i=3,9} B_{i} .
$$

Пусть

$$
\max _{1 \leqslant i \leqslant 9} B_{i}=\max _{i=1,7} B_{i}
$$

Для доказательства неравенства (3.1) достаточно доказать неравенства

$$
1+\frac{r}{r+2} \cdot B_{i} \leqslant T_{i}(i=1,7)
$$

т.е.

$$
1+r \sum_{k=1}^{3}\left|\alpha_{k i}\right| \leqslant\left|1-\alpha_{1 i}\right|+(1+r)\left|\alpha_{1 i}\right|+(2+r) \sum_{k=2}^{3}\left|\alpha_{k i}\right| .
$$

Так как $\left|1-\alpha_{1 i}\right|+(1+r)\left|\alpha_{1 i}\right| \geqslant 1-\left|\alpha_{1 i}\right|+(1+r)\left|\alpha_{1 i}\right|=1+r\left|\alpha_{1 i}\right|$, то остается доказать неравенство $r \sum_{k=2}^{3}\left|\alpha_{k i}\right| \leqslant(2+r) \sum_{k=2}^{3}\left|\alpha_{k i}\right|$, которое очевидно, так как $r<2+r$.

Случаи (3.3) доказываются аналогично.

Теорема 4. Оператор проектирования $\widetilde{\pi}_{\alpha}^{(0)}$ пространства $l_{\infty}^{9}$ на подпространство $\widetilde{Y}_{6}$, определяемое функционалами (2.3), является сильно единственным и максимальное значение константы сильной единственности $k_{0}$ равно $\frac{1}{2 r+1}$, если $0<r \leqslant \frac{1}{2}$. 
Доказательство. Снова от функционалов (2.3) перейдем к функционалам (2.4). Обозначив $s=1 / r$, легко заметить, что для функционалов (2.4) выполняется теорема 3. Тогда $k_{0}=\frac{s}{s+2}=\frac{1}{2 r+1}$, если $s \geqslant 2$ (или $0<r \leqslant \frac{1}{2}$ ).

Замечание 1. Вопрос о сильной единственности минимальных проекций с неединичной нормой, найденных в теоремах 1 и 2 при $0<r<2$ и $r>1 / 2$ соответственно, остается нерешенным.

\section{Заключение}

В работе найдены проекционные константы для минимальных операторов проектирования на на некоторый класс подпространств коразмерности 3 в пространстве $l_{\infty}^{9}$. Подпространства образованы с помощью гиперплоскостей рассматриваемого конечномерного пространства. Относительные проекционные константы вычислены как для минимальных проекций с единичной нормой, так и для минимальных проекций с нормой больше единицы. Максимальные значения констант сильной единственности найдены только для минимальных операторов проектирования с единичной нормой.

\section{Список литературы}

[1] Blätter J., Cheney E.W. Minimal projections on hyperplanes in sequence spaces // Annali di Matematica Pura ed Applicata. 1974. Vol. 101. Pp. 215-227.

[2] Bohnenblust H.F. Convex regions and projections in Minkowski spaces // Annals of Mathematics. 1938. Pp. 301-308.

[3] Chalmers B. L., Lewicki G. Three-dimensional subspace of with maximal projection constants // Journal of Functional Analysis. 2009. Vol. 257. Pp. 553592.

[4] König H.P., Lewis D.R., Lin P.-K. Finite dimensional projections // Studia Mathematica. 1983. Vol. 75, № 3. Pp. 341-358.

[5] Lewicki G. Best Approximation in Spaces of Bounder Linear Operators: Dissertationes Mathematicae. Warszawa: Instytut Matematyczny Polskiej Akademii Nauk, 1994. 103 p.

[6] Lewicki G., Micek A. Equality of two strong unique projection constants // Journal of Approximation Theory. 2010. Vol. 162, № 12. Pp. 2278-2289.

[7] Lokot' V.V. On a class of minimal projections in finite dimensional spaces // Optimization. 1994. Vol. 29. Pp. 311-317.

[8] Локоть В.В. Константы сильной единственности минимальных проекций на гиперплоскости в пространстве $l_{\infty}^{n}(n \geqslant 3) / /$ Математические заметки. 2002. T. 72, № 5. C. 723-728. https://doi.org/10.4213/mzm461 
[9] Локоть В.В., Мартынов О.М. Проекционные константы. Мурманск: МГГУ, 2013. 302 c.

[10] Martinov O.M. Constants of strong unicity of minimal projections onto some twodimensional subspaces of $l_{\infty}^{4} / /$ Journal of Approximation Theory. 2002. Vol. 118. Pp. 175-187.

[11] Мартынов О.М. Некоторые свойства операторов проектирования в банаховых пространствах: Диссертация к.ф.-м.н.. СПб: РГПУ им. А.И. Герцена, 2002.

[12] Мартынов О.М. Проекционные константы некоторого класса подпространств коразмерности два в пространстве $l_{\infty}^{2 n} / /$ Функциональный анализ и его приложения. 2019. Т. 53, № 3. С. 33-44.

[13] Мартынов О.М. О сильной единственности некоторых проекций с единичной нормой // Дифференциальные уравнения и процессы управления. 2020. Т. 2. C. 33-48.

[14] Newman D.J., Shapiro H.S. Some theorems on Chebyshev approximation // Duke Mathematical Journal. 1963. Vol. 30, № 4. Pp. 673-681.

[15] Newman D.J., Shapiro H.S. Approximation by Generalized Rational Functions // On Approximation Theory. Eds. by P.L. Butzer, J. Korevaar. Vol. 5. Basel: Springer, 1964. Pp. 245-251. https://doi.org/10.1007/978-3-0348-4131-3_25

[16] Одинец В.П. О семинаре по геометрии банаховых пространств в 1990-97 гг. // Некоторые актуальные проблемы современной математики и математического образования. Герценовские чтения 2007. T. LX. СПб: Изд-во БАН, 2007. C. $12-26$.

[17] Odyniec W., Lewicki G. Minimal Projections in Banach Spaces. Series: Lecture Notes in Mathematics. Vol. 1449. Berlin, New York: Springer, 1990.

[18] Odyniec W., Prophet M. The strong unicity constant and its applications // Banach Center Publications. 2008. Vol. 79, № 1. Pp. 167-172. http://dx.doi.org/10.4064/bc79-0-13

[19] Odyniec W., Prophet M.P. A lower bound of the strongly unique minimal projection constant of $l_{\infty}^{n},(n \geqslant 3) / /$ Journal of Approximation Theory. 2007. Vol. 145. Pp. 111-121.

[20] Одинец В.П., Якубсон М.Я. Проекторы и базисы в нормированных пространствах. М.: Едиториал УРСС, 2004.

\section{Образец цитирования}

Мартынов О.М. О сильной единственности минимальных проекций в пространстве $l_{\infty}^{9} / /$ Вестник ТвГУ. Серия: Прикладная математика. 2020. № 4 . С. 28-42. https://doi.org/10.26456/vtpmk603 


\section{Сведения об авторах}

\section{1. Мартынов Олег Михайлович}

доцент кафедры №13 военной академии воздушно-космической обороны имени Маршала Советского Союза Г.К. Жукова.

Россия, 170100, г. Тверь, ул. Жигарева, д.50, ВА ВКО им. Маршала Советского Союза Г.К. ЖКккова. E-mail: olegmartynov@yandex.ru 


\title{
ON THE STRONG UNIQUENESS OF MINIMAL PROJECTIONS IN THE SPACE $l_{\infty}^{9}$
}

\author{
Martynov Oleg Mikhailovich \\ Associate professor at department №13, \\ Zhukov Air and Space Defense Academy \\ Russia, 170100, Tver, Zhigarev Str., 50, Military Aerospace Defense Academy. \\ E-mail: olegmartynov@yandex.ru
}

Received 30.09.2020, revised 23.10.2020.

In this paper we consider minimal projections of the space $l_{\infty}^{9}$ on some subspaces of codimension 3. Relative projection constants are found for them, and in the case of a minimal projection with a unit norm, we find maximum value of the strong uniqueness constant.

Keywords: space, subspace, projection operator, relative projection constant, the constant of strong uniqueness.

\section{Citation}

Martynov O.M., "On the strong uniqueness of minimal projections in the space $l_{\infty}^{9} "$, Vestnik TvGU. Seriya: Prikladnaya Matematika [Herald of Tver State University. Series: Applied Mathematics], 2020, № 4, 28-42(in Russian). https://doi.org/10.26456/vtpmk603

\section{References}

[1] Blätter J., Cheney E.W., "Minimal projections on hyperplanes in sequence spaces", Annali di Matematica Pura ed Applicata, 101 (1974), 215-227.

[2] Bohnenblust H.F., "Convex regions and projections in Minkowski spaces", Annals of Mathematics, 1938, 301-308.

[3] Chalmers B. L., Lewicki G., "Three-dimensional subspace of with maximal projection constants", Journal of Functional Analysis, 257 (2009), 553-592.

[4] König H.P., Lewis D.R., Lin P.-K., "Finite dimensional projections", Studia Mathematica, 75:3 (1983), 341-358.

[5] Lewicki G., Best Approximation in Spaces of Bounder Linear Operators, Dissertationes Mathematicae, Instytut Matematyczny Polskiej Akademii Nauk, Warszawa, 1994, 103 pp.

[6] Lewicki G., Micek A., "Equality of two strong unique projection constants", Journal of Approximation Theory, 162:12 (2010), 2278-2289.

[7] Lokot' V.V., "On a class of minimal projections in finite dimensional spaces", Optimization, 29 (1994), 311-317. 
[8] Lokot' V.V., "Constants of Strong Uniqueness of Minimal Projections onto Hyperplanes in the Space $l_{\infty}^{n}(n \geqslant 3)$ ", Mathematical Notes, 72:5 (2002), 667-671, https://doi.org/10.4213/mzm461.

[9] Lokot V.V., Martynov O.M., Proektsionnye konstanty [Projection constants], MGGU, Murmansk, 2013 (in Russian), 302 pp.

[10] Martinov O.M., "Constants of strong unicity of minimal projections onto some two-dimensional subspaces of $l_{\infty}^{4} "$ ", Journal of Approximation Theory, 118 (2002), $175-187$.

[11] Martynov O.M., Nekotorye svojstva operatorov proektirovaniya v banakhovykh prostranstvakh, PhD Thesis, RGPU im. A.I. Gertsena, SPb, 2002 (in Russian).

[12] Martynov O.M., "Projection constants of a certain class of subspaces of codimension two in the space $l_{\infty}^{2 n}$ ", Funktsionalnyj analiz $i$ ego prilozheniya [Functional analysis and its applications], 53:3 (2019), 33-44 (in Russian).

[13] Martynov O.M., "On the strong uniqueness of some projections with unit norm", Differentsialnye uravneniya $i$ protsessy upravleniya [Differential equations and control processes], 2 (2020), 33-48 (in Russian).

[14] Newman D.J., Shapiro H.S., "Some theorems on Chebyshev approximation", Duke Mathematical Journal, 30:4 (1963), 673-681.

[15] Newman D.J., Shapiro H.S., "Approximation by Generalized Rational Functions", On Approximation Theory. V.5, eds. P.L. Butzer, J. Korevaar, Springer, Basel, 1964, 245-251, https://doi.org/10.1007/978-3-0348-4131-3_25.

[16] Odinets V.P., "On the seminar on the geometry of Banach spaces in 1990-97.", Nekotorye aktualnye problemy sovremennoj matematiki i matematicheskogo obrazovaniya. Gertsenovskie chteniya 2007 [Some actual problems of modern mathematics and mathematical education. Herzen Readings 2007]. V. LX, Izd-vo BAN, $\mathrm{SPb}, 2007,12-26$ (in Russian).

[17] Odyniec W., Lewicki G., Minimal Projections in Banach Spaces. V. 1449, Lecture Notes in Mathematics, Springer, Berlin, New York, 1990.

[18] Odyniec W., Prophet M., "The strong unicity constant and its applications", $B a$ nach Center Publications, 79:1 (2008), 167-172, http://dx.doi.org/10.4064/bc79$0-13$.

[19] Odyniec W., Prophet M.P., "A lower bound of the strongly unique minimal projection constant of $l_{\infty}^{n},(n \geqslant 3)$ ", Journal of Approximation Theory, 145 (2007), $111-121$.

[20] Odinets V.P., Yakubson M.Ya., Proektory i bazisy v normirovannykh prostranstvakh [Projectors and bases in normed spaces], Editorial URSS Publ., Moscow, 2004 (in Russian). 